\title{
Influence of rear-roof spoiler on the aerodynamic performance of hatchback vehicle
}

\author{
See-Yuan Cheng ${ }^{1,2,}$, and Shuhaimi Mansor $^{3}$ \\ ${ }^{1}$ Centre for Advanced Research on Energy, Universiti Teknikal Malaysia Melaka, Hang Tuah Jaya, \\ 76100 Durian Tunggal, Melaka, Malaysia \\ ${ }^{2}$ Faculty of Mechanical Engineering, Universiti Teknikal Malaysia Melaka, Hang Tuah Jaya, 76100 \\ Durian Tunggal, Melaka, Malaysia \\ ${ }^{3}$ Faculty of Mechanical Engineering, Universiti Teknologi Malaysia, 81310 UTM Skudai, Johor, \\ Malaysia
}

\begin{abstract}
Rear-roof spoiler is commonly used for improving the aerodynamic performance of road vehicles. This study aims to investigate the effect of strip-type rear-roof spoiler on the aerodynamic performance of hatchback vehicles. The main parameter of study was the inclination angle of the spoiler. A computational fluid dynamics (CFD) method was used. The numerically obtained results were compared to the experimental data for validation of the CFD method. The spoiler effectively reduced the aerodynamic lift at positive inclination angle by causing the surface pressure near the roof-spoiler junction to increase. However, its effect is unfavourable when configured at negative angle due to the downward accelerating flow that causes the surface pressure around the roof-spoiler junction to drop. Although the aerodynamic lift was found to decrease with the spoiler angle, this was accompanied by drag increment.
\end{abstract}

\section{Introduction}

A properly designed rear spoiler can reduce the rear-axle lift of a vehicle. This effect is important because a vehicle with its rear-axle lift lower than the front exhibits better stability, i.e. requires minimum driver intervention to maintain a straight path [1]. In addition, when a vehicle makes a turn, it needs sufficient traction to provide the centripetal force for it to pass the curve without slip. Hence, the use of spoiler will enable a vehicle to achieve greater downforce (negative lift), and thus better traction without significant increase in its weight.

There are two types of rear spoiler design: strips and free-standing wing. The effectiveness of wing-type spoiler has been reported in numerous studies. For instance, Tsai et al. [2] investigated numerically five different spoiler configurations. The spoilers are of inverted airfoil type and were mounted on the trunk of a simplified car model based on the HONDA S2000. Although all the cases had shown reduction in lift, however, only one out of five configurations succeeded in producing a negative lift coefficient value.

\footnotetext{
* Corresponding author: cheng@utem.edu.my
} 
Meanwhile, Daryakenari et al. [3] has shown a similar trends. Its numerical results indicate that up to $75 \%$ reduction in lift coefficient for a passenger car model can be achieved by properly manipulating the inclination angle of its flat-plate-type spoiler, i.e. at $50^{\circ}$ relative to the ground. Similarly, Kodali and Bezavada [4] has observed a decrease of $80 \%$ lift coefficient in the case of a simplified-passenger-car model mounted with an inverted-airfoil type spoiler.

As for strip-type rear spoilers, Menon et al. [5] has investigated the influence of spoiler on the aerodynamic drag and crosswind stability of a simplified hatchback model, namely, an Ahmed model at $35^{\circ}$ slant angle. Although these two performances are important, the study did not cover aerodynamic lift performance which is important for driving stability. Moreover, extensive literature search found that the coverage of this particular topic - striptype rear spoiler's influence on lift performance - is scarce. To fill the gap, hence, the main objective of the present paper is to investigate the effect of strip-type rear-roof spoiler on the aerodynamic lift performance of simplified hatchback model.

\section{Hatchback model and spoiler configurations}

The Ahmed body which represents simplified road vehicle geometry in the form of a bluff body was adopted. The slant angle was at $35^{\circ}$, which is typical for most hatchback cars. Figure 1 shows the Ahmed model fitted with a rear-roof spoiler configured at $0^{\circ}$ pitch angle. Also shown in the figure are the profiles of the spoiler at various pitch angles investigated in the present study, namely, $-15^{\circ}, 0^{\circ}, 5^{\circ}, 10^{\circ}$, and $15^{\circ}$. For all configurations, the length of the spoiler was fixed at $66.6 \mathrm{~mm}$, which corresponds to $30 \%$ of the length of the slant section. The trailing edge of the spoiler was filleted ( $5 \mathrm{~mm}$ radius) for avoiding highly skewed cells during meshing. For the same reason, the angle between the slant section and the rear face of the spoiler was maintained at right angle for all configurations. For details of the Ahmed model dimensions, the reader is referred to Ahmed [6].
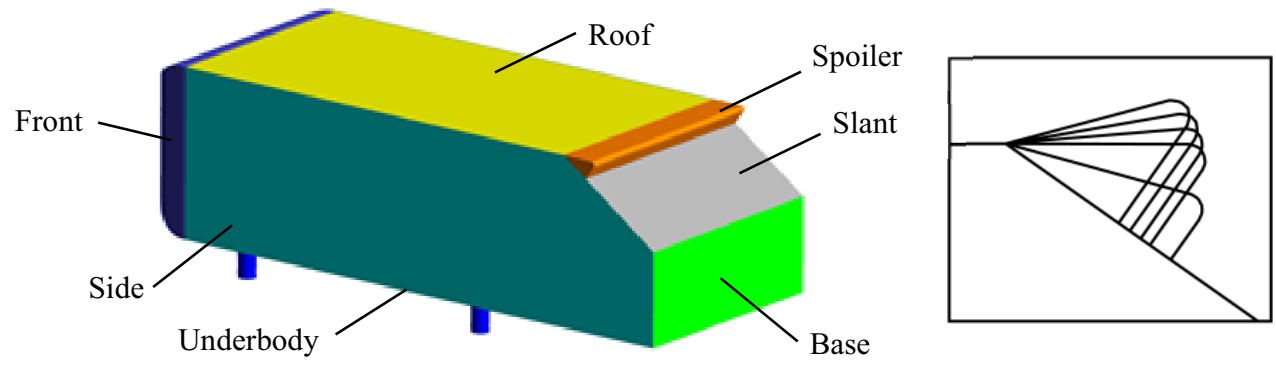

Fig. 1. Side view of Ahmed model fitted with a rear-roof spoiler (left), and the spoiler configurations (right).

\section{Computational method}

\subsection{CFD settings}

The present study employed a numerical simulation method to investigate the influence of pitch angle of rear spoiler on the aerodynamics forces of a simplified hatchback model. All results were obtained using the commercial finite-volume solver ANSYS Fluent 16. The Reynolds-averaged Navier-Stokes (RANS) approach was used. The governing equations solved in the simulations are the time-averaged continuity and momentum equations for an incompressible Newtonian fluid: 


$$
\begin{gathered}
\frac{\partial u_{i}}{\partial x_{i}}=0 \\
\rho u_{j} \frac{\partial u_{i}}{\partial x_{j}}=\frac{\partial}{\partial x_{j}}\left[-p \delta_{i j}+2 \mu S_{i j}-\rho u_{i}^{\prime} u_{j}^{\prime}\right]
\end{gathered}
$$

where, $u$ is the time-averaged velocity, $\rho$ is the fluid density, $p$ is the time-averaged static pressure, $\mu$ is the fluid viscosity, $S_{i j}$ is the mean rate of strain tensor, and the quantity $-\rho u_{i}^{\prime} u_{j}^{\prime}$ is the Reynolds stresses which represent the effects of turbulence. To close equation (2), the Reynolds stresses term must be modelled. The chosen turbulence model was the widely used two-equation model, namely, k-epsilon realizable model. The enhanced wall treatment (EWT) option of the simulation package was used. The EWT automatically imposes the wall function to model the boundary layer profile for estimation of the surface friction on the wall surfaces when the first grid point is in the log-layer range. The steady, pressure-based solver was utilized to achieve steady-state simulations. All reported results were obtained using a second-order node-based upwinding discretization scheme.

The inlet boundary condition was set as uniform flow with inlet velocity $U=40 \mathrm{~m} / \mathrm{s}$ and the turbulence intensities of $0.2 \%$. The corresponding Reynolds number (Re) was 768,000 based on the model height. As for the outlet boundary, the pressure outlet at zero gauge pressure was imposed. The side and top walls of the domain was defined as symmetry boundary condition. Meanwhile, the ground and model surfaces were set as noslip wall.

The computational domain resembles a rectangular box. Since the model is symmetric and the flow is steady, all simulation cases were run for half of the flow domain with a symmetry plane placed at the centreline location. The cross sectional area of the half flow domain was $1738 \mathrm{~mm} \times 1129.5 \mathrm{~mm}$ (height $\mathrm{x}$ width). The corresponding blockage ratio was less than $1.5 \%$, which is well within the typically accepted range of $5 \%$ in automotive aerodynamic testing [7]. The upstream and downstream extends of the domain were $1.4 l$ and $11.4 l$, respectively.

\subsection{Meshing}

The computational domain was decomposed into unstructured and prismatic cells (see Figure 2). The latter was employed around the model and the ground for improving the boundary layer resolution. The result of grid convergence study indicates that the mesh is sufficiently refined at around 315,000 nodes and 890,000 cells. The first prismatic cell layer thickness around the model surface was at $0.5 \mathrm{~mm}$. The corresponding $\mathrm{y}+$ ranges from around 1 to 57 . 


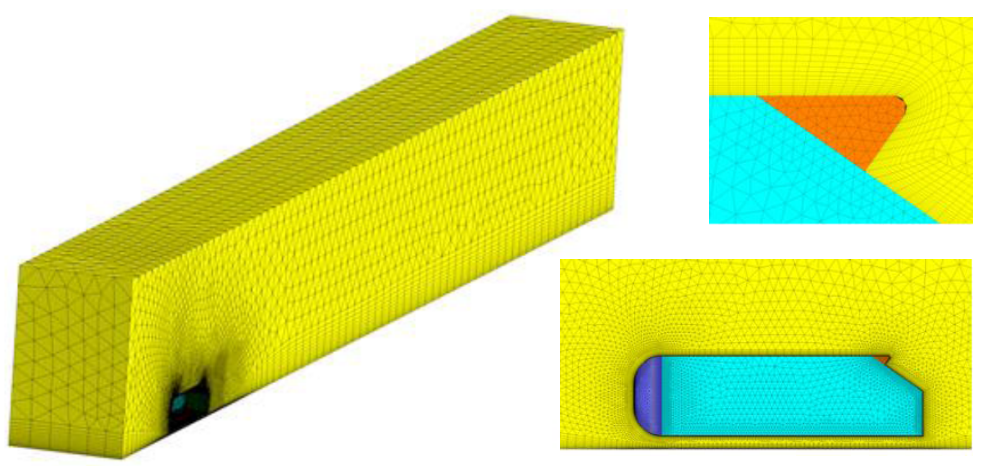

Fig. 2. Numerical cells of the simulation domain (left), close-up of prismatic cells around the rearroof spoiler (top right), and mesh density distribution around the Ahmed body (bottom right).

\subsection{Validation}

Validation of the numerical method was carried out by comparing the numerically obtained results of Ahmed model without the spoiler to the experimental results found in the literature. The Re was at 768,000 (based on the model height) which was consistent with the experimental Re of Lienhart et al. [8]. Figure 3 shows the flow features at the rear section of Ahmed model along the centreline reproduced by the present simulation. As depicted, the flow separates from the roof-backlight junction. It does not reattach at the slanted edge, but continues downstream and joins the separation bubble trailing the vertical base of the model. Qualitatively, this flow pattern is consistent with the experimental results reported in the literature (e.g. $[6,8,9])$.

Table 1 compares the drag coefficient $C_{d}$ values obtained from the present study to the experimental data by Lienhart et al. [8]. As shown, the total $C_{d}$ value was in excellent agreement with the experiment, i.e. percentage difference of $2.68 \%$. The drag breakdown shows that the $C_{d}$ of the base and slant sections were well predicted. Good prediction of $C_{d}$ in these two sections is important because the influence of the spoiler was expected to be most prominent at the rear section.

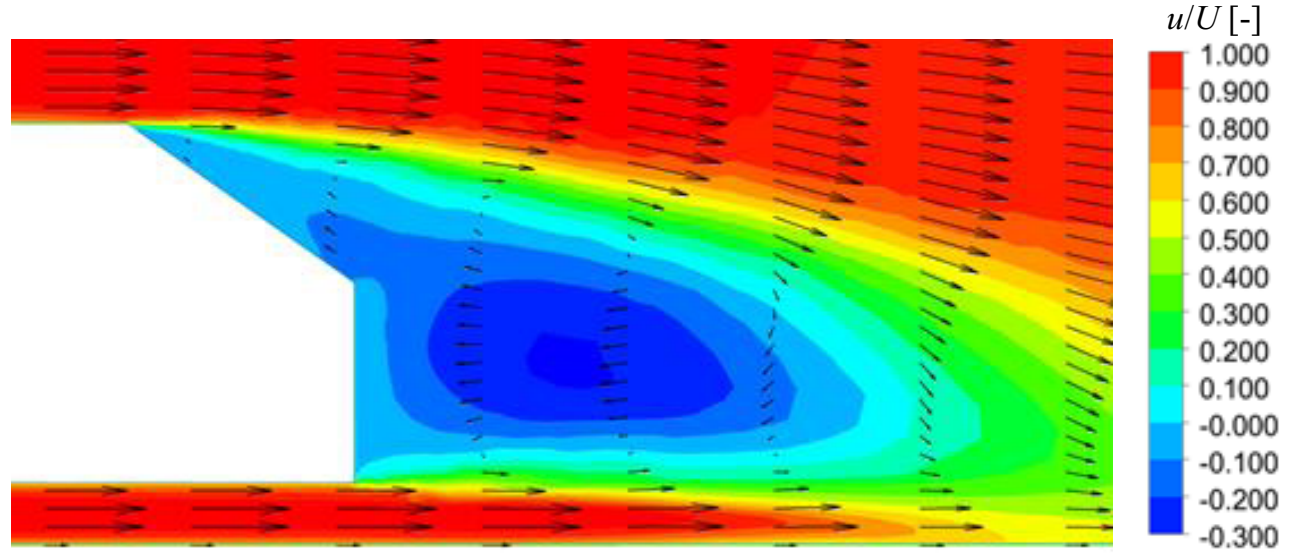

Fig. 3. Distribution of streamwise velocity component along the symmetry plane at the rear section of Ahmed model. 
Table 1. Comparison of the experimental $C_{d}$.

\begin{tabular}{|c|c|c|c|}
\hline Source & CFD & Experiment [8] & Percentage diff. (\%) \\
\hline Slant & 0.095 & 0.097 & 2.08 \\
\hline Base & 0.094 & 0.096 & 2.11 \\
\hline Front & 0.039 & 0.015 & 88.89 \\
\hline Viscous & 0.049 & 0.055 & 11.54 \\
\hline Total & 0.265 & 0.258 & 2.68 \\
\hline
\end{tabular}

\section{Results and discussion}

\subsection{Effects of rear spoiler angle}

Figure 4 shows that the lift coefficient $C_{l}$ decreased almost linearly with pitch angle for the range of pitch angle tested. In addition, it is evident that the benefit of fitting a spoiler only occurred at positive pitch angle. At negative pitch angle, relatively higher lift was found, which is deemed unfavourable to driving stability.

Figure 5 compares the distribution of static pressure coefficient $C_{p}$ (colour contour) and streamwise velocity component (vectors) around the rear section of the model at different spoiler configurations. In general, the flow at the upper body separated near the trailing edge of the roof when without the spoiler, whereas, it separated near the end of the spoiler for all spoiler angles when a spoiler is used. Besides, when a spoiler was configured at zero or positive pitch angle, it prevented the flow from accelerated near the roof-backlight junction. Thus, it resulted in a higher static pressure around the junction. As for the spoiler with negative pitch angle, augmentation of flow occurred near the junction, and was accompanied by significant drop in static pressure.

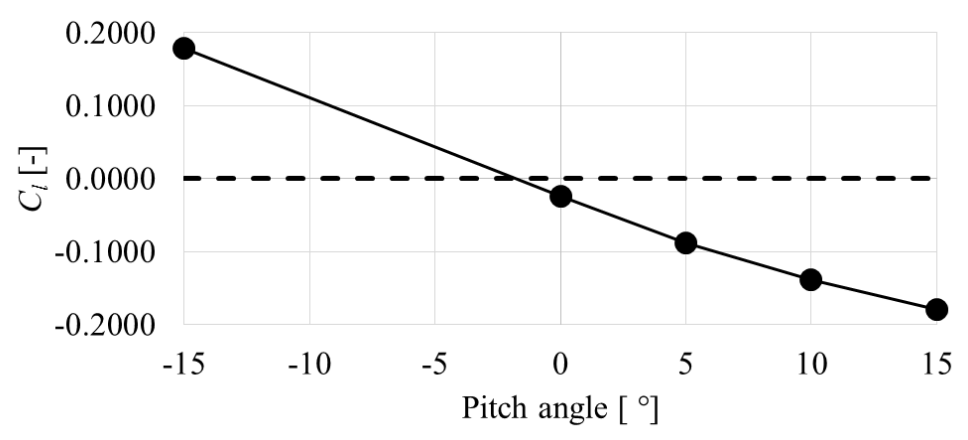

Fig. 4. Graph of $C_{l}$ against rear spoiler angle; Dashed-horizontal line is without spoiler.

Meanwhile, as depicted in Figure 5, the spoiler angle also has an effect on the size of the separation bubble behind the model. As shown, the height and length of the separation bubble are found to increase with larger spoiler angle.

Figure 6 compares the effect of spoiler angles on the $C_{p}$ distribution along the centreline of the model. As expected, the $C_{p}$ at the front section is almost identical in all cases. However, from the midsection onward, deviations in $C_{p}$ are gradually becoming more apparent along the upper body. The $0^{\circ}$ and $15^{\circ}$ cases exhibited higher $C_{p}$, with the latter being more pronounced, as compared to the case without a spoiler. Whilst, the $-15^{\circ}$ case shows a significant drop in $C_{p}$ particularly around the rear end of the roof, and the value peaked at the roof-spoiler junction. 


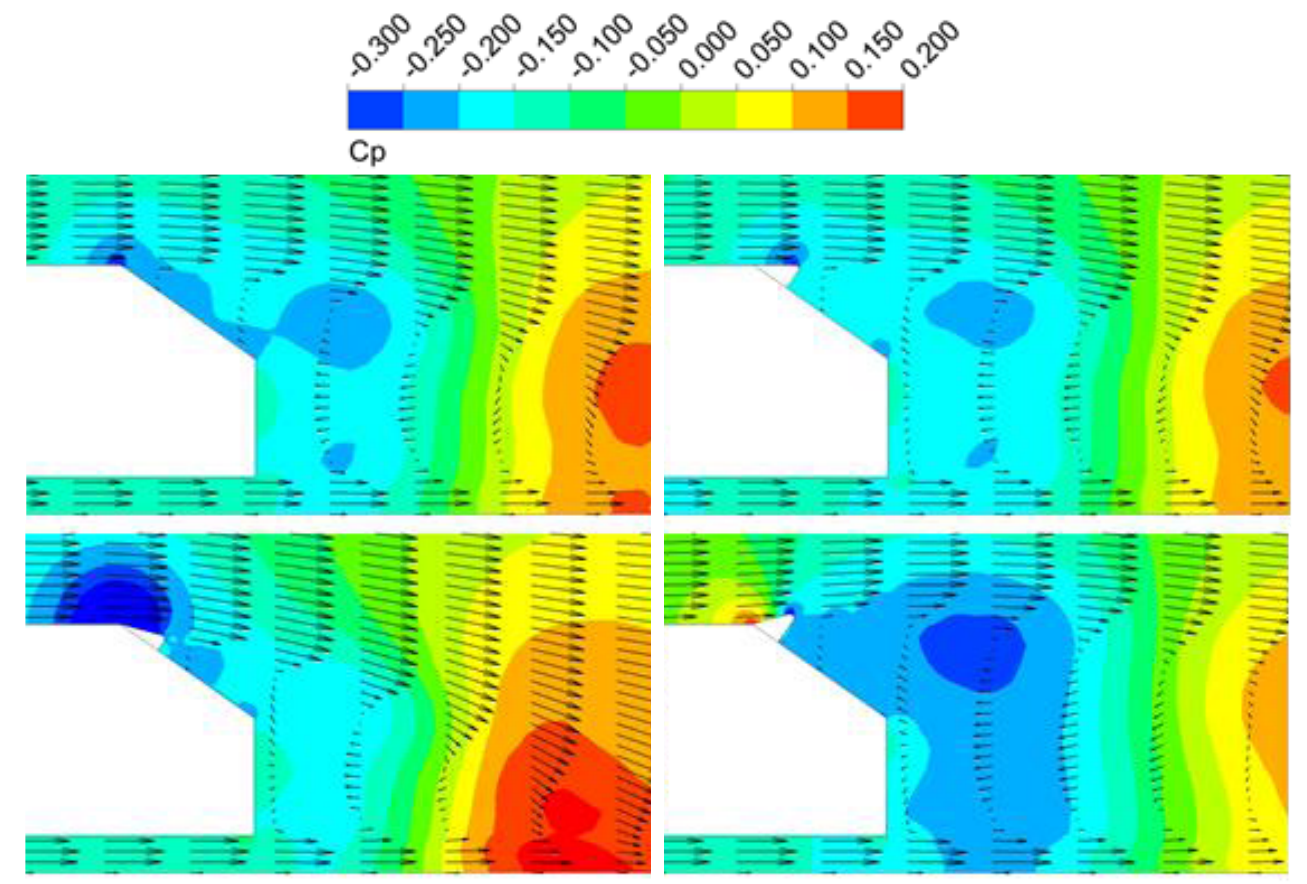

Fig. 5. Vectors of streamwise velocity component and $C_{p}$ distribution along the symmetry plane at the rear section of Ahmed model; Without spoiler (top left), with $0^{\circ}$ spoiler (top right), $15^{\circ}$ (bottom right), and $-15^{\circ}$ (bottom left).

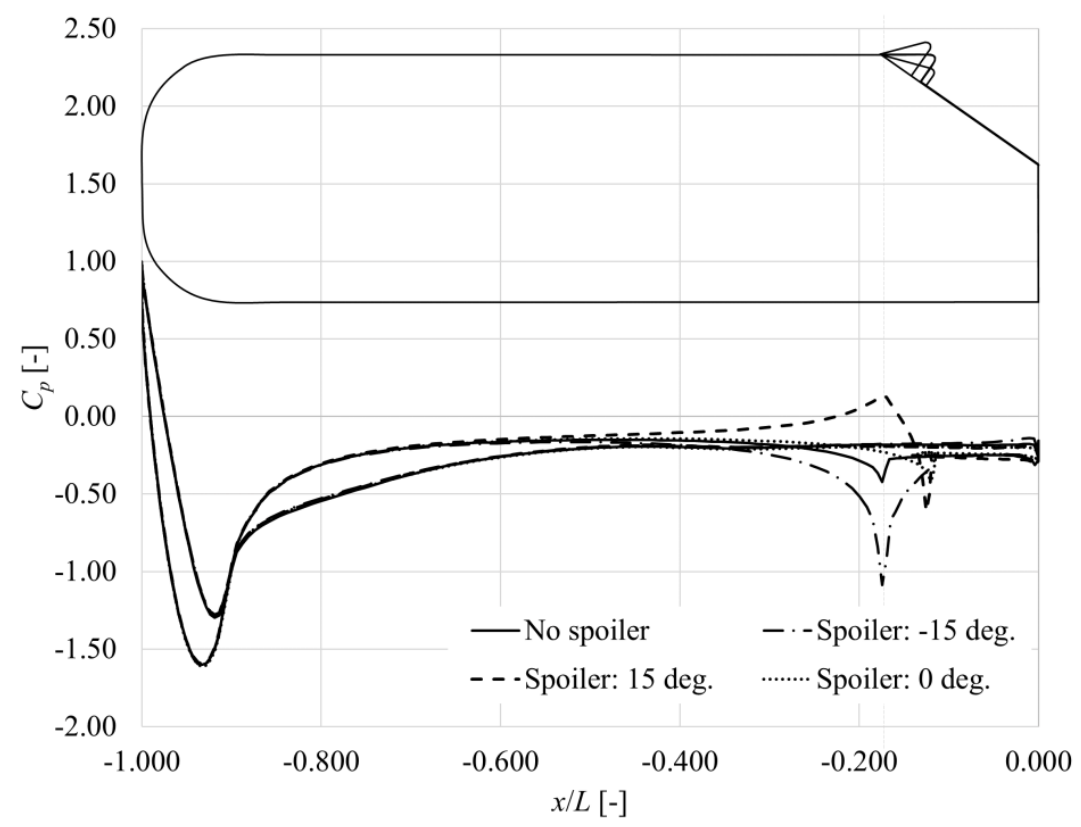

Fig. 6. $C_{p}$ distribution along the centerline of four cases: Without spoiler (solid curve), with $0^{\circ}$ spoiler (dotted curve), $15^{\circ}$ (dashed curve), and $-15^{\circ}$ (dashed-dotted curve). 
In general, the spoiler affects the aerodynamic lift of the model mainly by altering the flow near the rear end of the roof where the spoiler was mounted (see Figure 6 and 7). At $0^{\circ}$ inclination angle, the relatively higher surface pressure near the rear end of the roof has caused significant reduction in $C_{l}$ (by about $488 \%$ ) as compared to the case without the spoiler. In addition, the $C_{d}$ has reduced by about 3\% (see Figure 8 ). Note that this positive combination - reduction in both the drag and lift - is highly appreciable as it is often the case that the element that increases the downforce will also increase drag (e.g. [2-4]).

At $15^{\circ}$ pitch angle, significant increase in the surface pressure near the roof-spoiler junction may be evident (see Figure 7). This high pressure region has resulted in the dramatic drop in $C_{l}$, by about $2937 \%$. However, it was accompanied by about $11 \% C_{d}$ increment. Hence, this configuration might not be suitable in situations where fuel economy is important.

At negative inclination angle (i.e. the $-15^{\circ}$ case), the airflow around the roof-spoiler junction accelerated downward with the corresponding drop in the model's surface pressure around the region. Therefore, the $C_{l}$ has increased (by about 2737\%).

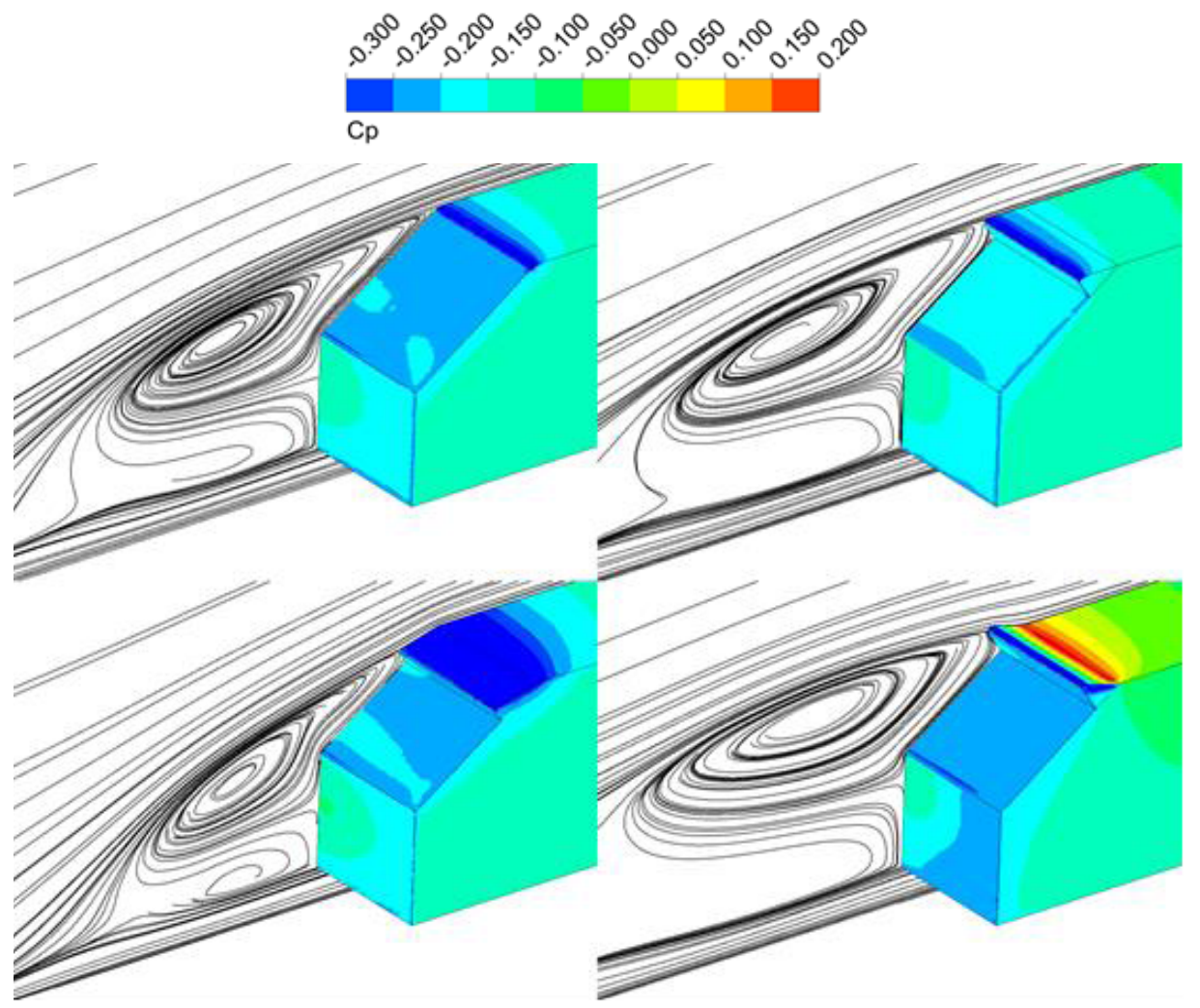

Fig. 7. Surface pressure distribution around the rear section of the model and the streamlines along the symmetry plane; Without spoiler (top left), with $0^{\circ}$ spoiler (top right), $15^{\circ}$ (bottom right), and $-15^{\circ}$ (bottom left).

Figure 8 shows that in general, the reduction in lift is accompanied by drag penalty. Therefore, lift reduction practice has to be carried out with care so as not to introduce significant drag increment in cases where drag performance is important such as in hybrids and EVs. This could be the reason why the use of rear-roof spoiler at high pitch angle (above $5^{\circ}$ ) is scarce in commercial vehicles as their need for downforce is not as crucial as in race cars. 


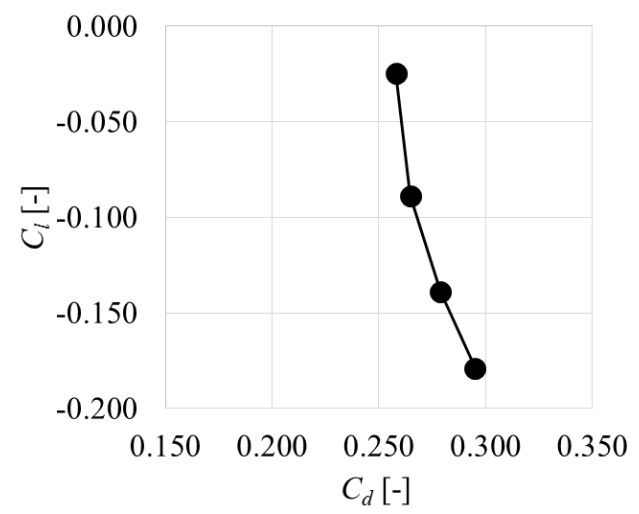

Fig. 8. Graph of $C_{l}$ against $C_{d}$ of the model fitted with the strip-type rear-roof spoiler.

\subsection{Body part contribution to $C_{l}$ reduction}

Figure 9 compares the percentage change of $C_{l}$ per body part as the pitch angle of the spoiler increases from $0^{\circ}$ to $5^{\circ}$, and from $5^{\circ}$ to $15^{\circ}$. As depicted, the decrease in $C_{l}$ was mainly come from the spoiler and roof. This result supports the qualitative discussions pertaining to Figure 7.

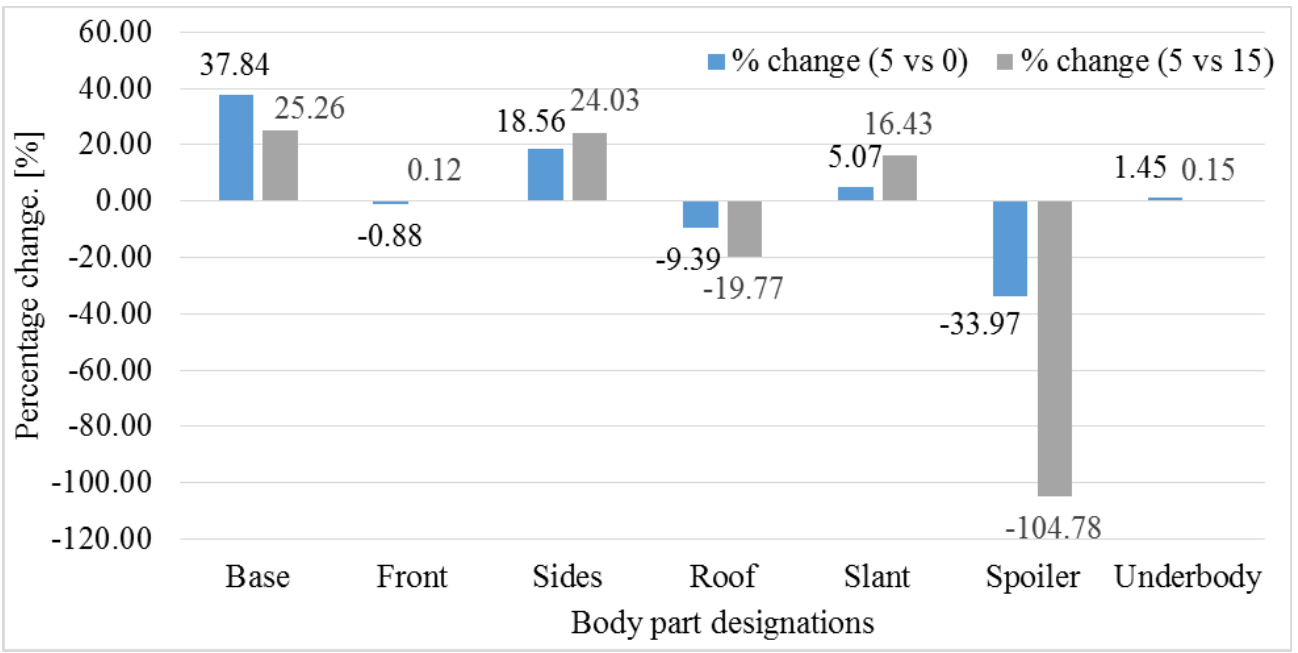

Fig. 9. Percentage change in $C_{l}$ per body part as the pitch angle of the spoiler increases from $0^{\circ}$ to $5^{\circ}$ (left bar), and from $5^{\circ}$ to $15^{\circ}$ (right bar).

\section{Conclusions}

This paper investigated the effect of strip-type rear-roof spoiler on the aerodynamic performance of hatchback vehicles by a RANS-based CFD method. The results show that the aerodynamic lift was to decrease nearly linearly with the inclination angle of the spoiler, and was accompanied by the increment in aerodynamic drag. However, it is possible to achieve reduction in both the lift and drag when the spoiler is configured at $0^{\circ}$ - parallel to the roof. In contrast, at negative spoiler angle, the use of rear spoiler was counterproductive to both the lift and drag reductions. In addition, when a spoiler is used, apart from the 
spoiler itself, the main body part that has contributed to the lift reduction was the model's roof, particularly at the rear end where the spoiler was mounted.

The present results were obtained from stationary simulations in which the motion of vehicle body was not considered. In practice, motion of vehicle body is very common and could change the inclination angle of the spoiler when the motion mode is of pitching. Hence, it helps to provide insight into how each spoiler configurations would perform under a more realistic driving condition if certain motion mode could be incorporated in the flow simulation. For this purpose, the method introduced by Cheng et al. $[10,11]$ could be employed in the future studies.

The authors would like to thank Universiti Teknikal Malaysia Melaka (UTeM) and Ministry of Higher Education for supporting this research under FRGS FRGS/1/2015/TK03/FKM/02/F00273.

\section{References}

1. J. Howell, and G. Le Good, The influence of aerodynamic lift on high speed stability, SAE Paper No 1999-01-0651 (1999)

2. C. H. Tsai, L. M. Fu, C. H. Tai, Y. L. Huang, and J. C. Leong, Computational aeroacoustic analysis of a passenger car with a rear spoiler, J. Appl. Math. Model. 33, No. 9, pp. 3661-3673 (2009)

3. B. Daryakenari, S. Abdullah, R. Zulkifli, E. Sundararajan, and A. Mohd Sood, Numerical study of flow over ahmed body and a road vehicle and the change in aerodynamic characteristics caused by rear spoiler, Int. J. Fluid Mech. Res. 40(4), pp. 354-372 (2013)

4. S. P. Kodali, and S. Bezavada, Numerical simulation of air flow over a passenger car and the influence of rear spoiler using CFD, IJATP, 01(1), pp. 6-13 (2012)

5. D. P. Menon, G. S. Kamat, Y. S. Mukkamala, and P. S. Kulkarni, To improve the aerodynamic performance of a model hatchback car with the addition of a rear roof spoiler, 16th Annual CFD Symposium, August 11-12, Bangolore (2014)

6. S. R. Ahmed, An experimental study of the wake structures of typical automobile shapes, J. Wind Eng. Ind. Aerodyn, 9(1-2), pp. 49-62 (1981)

7. W-H. Hucho, and G. Sovran, Aerodynamics of road vehicles, Annu. Rev. Fluid Mech, 25, pp. $485-537$ (1993)

8. H. Lienhart, C. Stoots, and S. Becker, Flow and turbulence structures on the wake of a similified car model (Ahmed model), DGLR Fach. Symp. der AG ATAB, Stuttgart University (2000)

9. G. Vino, S. Watkins, P. Mousley, J. Watmuff, and S. Prasad, Flow structures in the near-wake of the Ahmed model, J Fluid Struct, 20(5), pp. 673-695 (2005)

10. S.Y. Cheng, M. Tsubokura, T. Nakashima, T. Nouzawa, Y. Okada, A numerical analysis of transient flow past road vehicles subjected to pitching oscillation, J Wind Eng Ind Aerod, 99, pp. 511-522 (2011)

11. S.Y. Cheng, M. Tsubokura, T. Nakashima, Y. Okada, T. Nouzawa, Numerical quantification of aerodynamic damping on pitching of vehicle-inspired bluff body, $\mathrm{J}$ Fluid Struct, 30, pp. 188-204 (2012) 